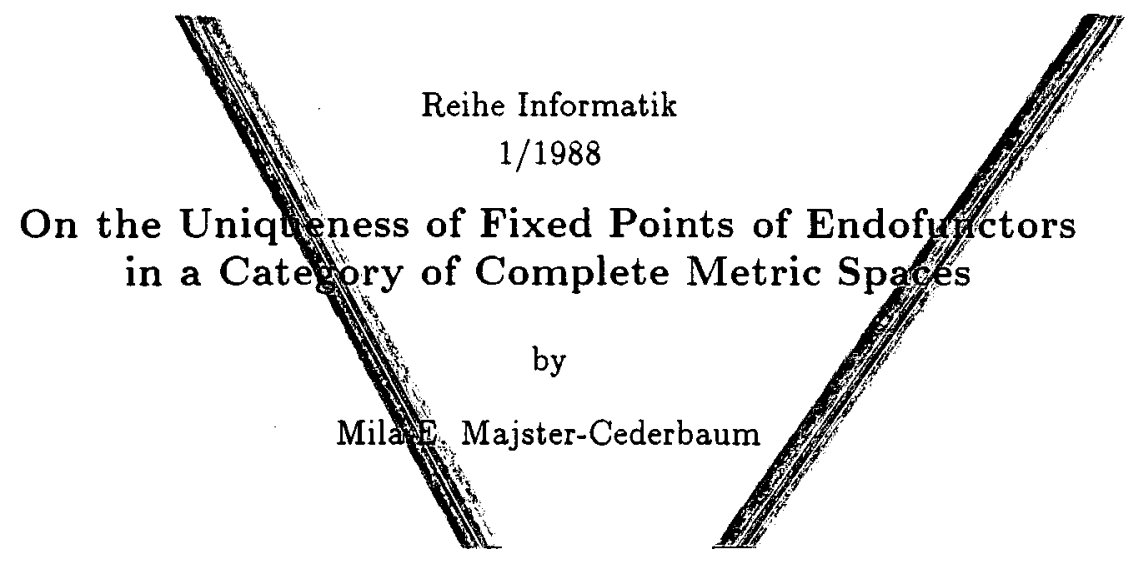

March 1988

\title{
Summary
}

In [S] de Bakker and Zucker proposed to use complete metric spaces for the semantic definition of program. ming languages that allow for concurrency and synchronisation. The use of the tools of metric topology has been advocated by Nivat and his colleagues already in the seventies and metric topology was successfully applied to various problems [11,12]. Recently, the question under which cricumstances fixed point equations involuing complete metric spaces can be (uniguely) solved has attracted attention, e.g. [1, 10]. In [1], a criterion for the existence of a solution, namely the contractiveness of the respective functor, is provided. Contractiveness together with an additional criterion, the hom-contractiveness was shown in [1] to guarantee uniqueness. The problem of uniqueness is the topic of our contribution. 


\section{Mathematical Preliminaries}

A metric space is a pair $(M, d)$ with $M$ a set and $d$ a mapping, $d: M \times M \rightarrow[0,1]$ which satisfies (1)

(a) $\forall x, y \in M \quad(d(x, y)=0 \Leftrightarrow x=y)$,

(b) $\forall x, y \in M \quad d(x, y)=d(y, x)$,

(c) $\forall x, y, z \in M \quad d(x, y) \leq d(x, z)+d(z, y)$.

A sequence $\left(x_{i}\right)$ in a metric space $(M, d)$ is a Cauchy sequence, whenever $\forall \epsilon>0$ $\exists N \in \mathbb{N} \forall n, m>N \quad d\left(x_{n}, x_{m}\right)<\epsilon$. The metric space $(M, d)$ is called complete if every Cauchy sequence converges to an element of $M$

Let $\left(M_{1}, d_{1}\right),\left(M_{2}, d_{2}\right)$ be metric spaces. A function $f: M_{1} \rightarrow M_{2}$ is called non-distance-increasing, if $\forall x, y \in M_{1}$

$$
d_{2}(f(x), f(y)) \leq d_{1}(x, y) .
$$

$f$ is called (isometric) embedding, if $\forall x, y \in M_{1}$

$$
d_{2}(f(x), f(y))=d_{1}(x, y) .
$$

$f$ is called an isometry, if $f$ is onto and an (isometric) embedding.

It is well known, [4], that every metric space $(M, d)$ can be embedded into a "unique" "minimal" complete metric space, called the completion of $(M, d)$.

Let $M$ denote the category that has metric spaces as objects and non-distance-increasing functions as arrows. Let $\mathbf{C}$ denote the category that has complete metric spaces as objects. The arrows in $\mathrm{C}$ are the non-distance-increasing functions. Let $M_{1}, M_{2}$ be complete metric spaces, and let

$$
i: M_{1} \rightarrow M_{2} \text { be an embedding }
$$

and $j: M_{2} \rightarrow M_{1}$ be a non-distance-increasing function.

$j$ is called a cut for $i$ if $i \circ j=i d_{M_{1}}{ }^{(2)}$. For embedding $i: M_{1} \rightarrow M_{2}$ with cut $j$ we put $\iota=(i, j)$ and write $M_{1} \longrightarrow^{\imath} M_{2}$ and define

$$
\underline{\delta(\iota)}=\sup _{x \in M_{2}}\left\{d_{M_{2}}(x, i(j(x)))\right\} \text {. }
$$

We say that a functor $\mathcal{F}: \mathrm{C} \rightarrow \mathrm{C}$ preserves embeddings iff $\mathcal{F} e$ is an embedding for every embedding $e$. A functor $\mathcal{F}: \mathbf{C} \rightarrow \mathbf{C}$ that preserves embeddings is called contracting if there exists an $\epsilon, 0 \leq \epsilon<1$, such that for all $D \longrightarrow^{\iota} E \in \mathrm{C}, \iota=(i, j)$,

$$
\delta(\mathcal{F} \iota) \leq \epsilon \cdot \delta(\iota)
$$

where $\mathcal{F}_{\iota}=\left(\mathcal{F}_{i}, \mathcal{F} j\right)$.

Please note that we have modified the definition of [1] slightly, just in order to be able to include the empty space as an object.

(1) $0 \leq d(x, y) \leq 1$ can be always obtained for an arbitrary metric $\widehat{d}: M \times M \rightarrow \mathbb{R}$ by substituting $\widehat{d}(x, y)$ by $\frac{\hat{d}(x, y)}{\hat{d}(x, y)+1}$.

(2) Throughout this text the composition $f \circ g$ of functions stands for $\lambda x . g(f(x))$. 


\section{The problem setting}

In [3] de Bakker et al. proposed a promising approach to the definition of semantics for programming languages based on complete metric spaces as follows: the meaning function $M e$ maps a program $P$ (in a language $\mathcal{L}$ ) to its meaning $M e[P]$ which is an element of a certain complete metric space constructed for the language $\mathcal{L}$ as a solution of a certain fixed point equation $\mathcal{F} X=X$.

This technique has been successfully applied $[3,17]$ e.g. to different variants of Hoare's communicating sequential processes [8]. Ever since, the question of existence and uniqueness of solutions of such fixed point equations as well as the connection to other models for semantics, e.g. the denotational approach based on CPO's (complete partial orders) $[5,13,14,15,16]$, has attracted interest. The latter question has been partly investigated in $[6,9,17]$. Recently, $[1,10]$ have developed general criteria to ensure existence and uniqueness of solutions of equations $\mathcal{F} X=X$, where $\mathcal{F}$ is an endofunctor in a category of complete metric spaces. In particular in [10] two different criteria, each of which guarantees the existence and uniqueness of fixed points are presented. In addition in [10] an interesting example of a fixed point equation is discussed that cannot be solved by standard techniques. In [1] it is shown that a functor that is contracting (as defined above) has a fixed point. It is then shown in [1] that contractiveness together with hom-contractiveness guarantees that the fixed point is unique (up to isometry). Here we discuss the problem of uniqueness. 


\section{Fixpoints of contracting functors}

In this section we deal with the problem how the fixed points of a contracting functor are related. In particular we show that every contracting functor has a unique "minimal" fixed point and that every contracting functor $\mathcal{F}$ with $|\mathcal{F} \emptyset|=1$ has a fixed point that is unique up to isometry.

\section{Remark 1}

Let $(M, d)$ be a complete metric space. Let

$$
\wp_{c}(M)=\{U \subset M: U \text { is non-empty and closed }\}
$$

and let $\tilde{d}_{M}$ denote the Hausdorff metric on $\wp_{c}(M)$, i.e. for $x, y \in M, X, Y \in \wp_{c}(M)$ let

$$
\begin{aligned}
\Delta(x, Y) & =\inf _{y \in Y} d(x, y) \\
\tilde{d}_{M}(X, Y) & =\max \left\{\sup _{x \in X}\{\Delta(x, Y)\}, \sup _{y \in Y}\{\Delta(y, X)\}\right\} .
\end{aligned}
$$

It is well-known that $\left(\wp_{c}(M), \tilde{d}_{M}\right)$ is a complete metric space, see e.g. [4].

Let now $D \longrightarrow \iota E \in \mathrm{C}, \iota=(i, j)$. In particular $D$ is isometrically embedded into $E$ via $i$ and we can view $D$ as a closed subset of $E$, i.e. an element of $\wp_{c}(E)$. Let $\tilde{d}_{E}$ be the Hausdorff metric on $\wp_{c}(E)$ then we can talk about

$$
\tilde{d}_{E}(D, E)
$$

or

$$
\widetilde{d}(D, E)
$$

for ease of notation, considering $D$ and $E$ as elements of $\wp_{c}(E)$.

\section{Remark 2}

Let $D \longrightarrow \iota \in \mathbf{C}, \iota=(i, j)$. If $\forall x \in E$

$$
d_{E}(x, i(j(x))) \leq \mu
$$

then

$$
\widetilde{d}(D, E) \leq \mu .
$$

This can be easily seen by the definition of the Hausdorff metric.

(3) Strictly speaking the "distance of $D$ and $E$ as elements of $\wp_{c}(E)$ " also depends on the choice of the embedding $i$. But as this choice will always be clear from the context, we omit its indication. 


\section{Remark 3}

Let $D \longrightarrow^{i} E \in \mathrm{C}$ then

$$
\tilde{d}(D, E) \leq \delta(\iota)
$$

by the above remark and the definition of $\delta(\iota)$.

\section{Theorem}

Let $\mathcal{F}: \mathrm{C} \rightarrow \mathrm{C}$ be a contracting functor and let $\emptyset$ denote the empty space.

a) There is a fixed point $M_{0}$ of $\mathcal{F}$ that can be embedded into any other fixed point $M$ of $\mathcal{F}$.

b) if $|\mathcal{F} \emptyset|=1$ then there is a unique fixed point of $\mathcal{F}$ up to isometry.

\section{Proof}

a) If $\mathcal{F} \emptyset=\emptyset$ the statement is trivial. Let now $\mathcal{F} \emptyset \neq \emptyset$. Clearly $\emptyset$ is initial in the categories $\mathbf{M}$ and $\mathbf{C}$. In particular there is a unique embedding

$$
\lambda_{0}: \emptyset \rightarrow \mathcal{F} \emptyset \text {. }
$$

By iteration we get $\lambda_{i}=\mathcal{F}^{i}\left(\lambda_{0}\right): \mathcal{F}^{i} \emptyset \rightarrow \mathcal{F}^{i+1} \emptyset, i \geq 1$, is an embedding. Let $\bigcup \mathcal{F}^{i} \emptyset$ denote the direct limit of this sequence in $M$. Then the completion of $\cup \mathcal{F}^{i} \emptyset$, which we denote by $\overline{U \mathcal{F}^{i} \emptyset}$, is the direct limit of the sequence in $\mathbf{C}$. Let $l_{i}: \mathcal{F}^{i} \emptyset \rightarrow \overline{\bigcup \mathcal{F}^{i} \emptyset}$ be the canonical embeddings, $i \geq 0$, i.e.

$$
l_{i}=\lambda_{i} \circ l_{i+1}
$$

Let us now choose an arbitrary one element space $\left\{x_{0}\right\}$. Clearly there is an embedding

$$
e_{0}:\left\{x_{0}\right\} \rightarrow \mathcal{F} \emptyset
$$

as $\mathcal{F} \emptyset \neq \emptyset$. Moreover, there is a unique embedding

$$
i_{0}: \emptyset \rightarrow\left\{x_{0}\right\}
$$

and we have

$$
\lambda_{0}=i_{0} \circ e_{0}
$$

as $\emptyset$ is initial. Let now $\sigma_{i}: \mathcal{F}^{i}\left\{x_{0}\right\} \rightarrow \mathcal{F}^{i+1}\left\{x_{0}\right\}, i \geq 0$, be defined as

$$
\begin{aligned}
\sigma_{0} & =e_{0} \circ \mathcal{F} i_{0} \\
\sigma_{i} & =\mathcal{F} \sigma_{i-1}, \quad i \geq 1 .
\end{aligned}
$$

Clearly, $\sigma_{i}$ is an embedding from $\mathcal{F}^{i}\left\{x_{0}\right\}$ to $\mathcal{F}^{i+1}\left\{x_{0}\right\}$. Let $\bigcup \mathcal{F}^{i}\left\{x_{0}\right\}$ denote the direct limit in $\mathbf{M}$ with respect to $\left\{\sigma_{i}\right\}$.

We are now going to embed $\mathcal{F}^{i}\left\{x_{0}\right\}$ into $\overline{\mathcal{U F}^{i} \emptyset}$ in a way that is compatible with $\sigma_{i}$. Let in particular

$$
\begin{gathered}
e_{0} \circ l_{1}:\left\{x_{0}\right\} \rightarrow \overline{\bigcup \mathcal{F}^{i \emptyset}} \\
\mathcal{F}^{i} e_{0} \circ l_{i+1}: \mathcal{F}^{i}\left\{x_{0}\right\} \rightarrow \overline{\bigcup \mathcal{F}^{i \emptyset}} \quad \text { for } i \geq 1 .
\end{gathered}
$$


These embeddings of $\mathcal{F}^{i}\left\{x_{0}\right\}$ into $\overline{U \mathcal{F}^{i} \emptyset}$ are compatible with the embedding $\sigma_{i}: \mathcal{F}^{i}\left\{x_{0}\right\} \rightarrow \mathcal{F}^{i+1}\left\{x_{0}\right\}$, as

$$
e_{0} \circ l_{1}=\sigma_{0} \circ\left(\mathcal{F} e_{0} \circ l_{2}\right)
$$

by equations $(I),(I I),(I I I)$. And in general

$$
\begin{aligned}
\mathcal{F}^{i} e_{0} \circ l_{i+1} & =\mathcal{F}^{i} e_{0} \circ \lambda_{i+1} \circ l_{i+1} \quad \text { by }(I) \\
& =\mathcal{F}^{i} e_{0} \circ \mathcal{F}^{i+1} \lambda_{0} \circ l_{i+2} \quad \text { by } \text { Def. } \\
& =\mathcal{F}^{i} e_{0} \circ \mathcal{F}^{i+1} i_{0} \circ \mathcal{F}^{i+1} e_{0} \circ l_{i+2} \quad \text { by }(I I) \\
& =\mathcal{F} \sigma_{0} \circ \mathcal{F}^{i+1} e_{0} \circ l_{i+2} \quad \text { by }(I I I) \\
& =\sigma_{i} \circ \mathcal{F}^{i+1} e_{0} \circ l_{i+2} \quad \text { by } \text { Def. }
\end{aligned}
$$

As $\overline{\cup \mathcal{F}^{i}\left\{x_{0}\right\}}$ is the direct limit with respect to the $\left\{\sigma_{i}\right\}$ we conclude that there is a unique embedding

$$
e: \overline{\mathcal{F}^{i}\left\{x_{0}\right\}} \rightarrow \overline{\mathcal{J F}^{i} \emptyset}
$$

such that

$$
h_{i} \circ e=\mathcal{F}^{i} e_{0} \circ l_{i+1}
$$

where $h_{i}$ is the canonical embedding of $\mathcal{F}^{i}\left\{x_{0}\right\}$ into $\overline{\cup \mathcal{F}^{i}\left\{x_{0}\right\}}, i \geq 0$.

Let now $N$ be an arbitrary fixed point of $\mathcal{F}$ and let $h: \mathcal{F} N \rightarrow N$ be an isometry. Cleraly, there is a unique embedding

$$
\epsilon_{0}: \emptyset \rightarrow N
$$

hence

$$
\begin{aligned}
& \epsilon_{i}: \mathcal{F}^{i} \emptyset \rightarrow N, \quad i \geq 1 \\
& \epsilon_{i}=\mathcal{F} \epsilon_{i-1} \circ h
\end{aligned}
$$

are embeddings of $\mathcal{F}^{i} \emptyset$ into $N$. We claim that they are compatible with the $\lambda_{i}$. First we observe that

$$
\begin{aligned}
\epsilon_{0} & =\lambda_{0} \circ \mathcal{F} \epsilon_{0} \circ h \\
& =\lambda_{0} \circ \epsilon_{1} \quad \text { by the initiality of } \emptyset
\end{aligned}
$$

and assuming $\epsilon_{i}=\lambda_{i} \circ \epsilon_{i+1}$ we get

$$
\begin{aligned}
\epsilon_{i+1} & =\mathcal{F} \epsilon_{i} \circ h \\
& =\mathcal{F}\left(\lambda_{i} \circ \epsilon_{i+1}\right) \circ h \\
& =\mathcal{F} \lambda_{i} \circ \mathcal{F} \epsilon_{i+1} \circ h \\
& =\lambda_{i+1} \circ \epsilon_{i+2} \quad \text { by Definition. }
\end{aligned}
$$


Again, as $\overline{U \mathcal{F}^{i} \emptyset}$ is the direct limit with respect to $\left\{\lambda_{i}\right\}$ we conclude that there is a unique embedding

$$
f: \overline{\bigcup \mathcal{F}^{i} \emptyset} \rightarrow N
$$

such that

$$
\epsilon_{i}=l_{i} \circ f
$$

hence

$$
e \circ f: \overline{\bigcup \mathcal{F}^{i}\left\{x_{0}\right\}} \rightarrow N \text { is an embedding. }
$$

We now construct cuts $c_{i}$ for $\sigma_{i}$ by

$$
\begin{aligned}
& c_{0}=\lambda x x_{0}, \quad c_{0}: \mathcal{F}\left\{x_{0}\right\} \rightarrow\left\{x_{0}\right\} \\
& c_{i}=\mathcal{F} c_{i-1}, \quad i \geq 1
\end{aligned}
$$

If $\mathcal{F}$ is contracting it has been shown in $[1,10]$ that $\overline{\cup \mathcal{F}^{i}\left\{x_{0}\right\}}$ is a fixed point of $\mathcal{F}$, hence we put $M_{0}=\overline{\mathcal{J F}^{i}\left\{x_{0}\right\}}$ to get the desired result.

b) Let now in addition $|\mathcal{F} \emptyset|=1$. Let $\mathcal{F} \emptyset=\left\{y_{0}\right\}$. We consider again the sequence $\mathcal{F}^{i} \emptyset, i \geq 0$, with embeddings $\lambda_{i}, i \geq 0$, as defined in a). We define a cut for $\lambda_{i}$, $i \geq 1$, by

$$
\begin{aligned}
& d_{1}=\lambda x \cdot y_{0} \\
& d_{i}=\mathcal{F} d_{i-1}, \quad i \geq 2
\end{aligned}
$$

Let now as in a) $N$ be another fixed point of $\mathcal{F}$ with the embeddings $\epsilon_{i}: \mathcal{F}^{i} \emptyset \rightarrow N$ defined in (VI), that are compatible with the $\lambda_{i}$ (see (VIII)). Let

$$
\begin{aligned}
& g_{1}=\lambda x . y_{0} \quad g_{1}: N \rightarrow\left\{y_{0}\right\} \\
& g_{i}=h^{-1} \circ \mathcal{F} g_{i-1} \quad g_{i}: N \rightarrow \mathcal{F}^{i} \emptyset
\end{aligned}
$$

where $h: \mathcal{F} N \rightarrow N$ is isometry. Clearly $g_{1}$ is a cut for $\epsilon_{1}$, and consequently

$$
\begin{aligned}
\epsilon_{i} \circ g_{i} & =\left(\mathcal{F} \epsilon_{i-1} \circ h\right) \circ\left(h^{-1} \circ \mathcal{F} g_{i-1}\right) \\
& =\mathcal{F}\left(\epsilon_{i-1} \circ g_{i-1}\right) .
\end{aligned}
$$

Clearly $\delta\left(\left(\epsilon_{1}, g_{1}\right)\right) \leq 1$ and

$$
\begin{aligned}
\delta\left(\left(\epsilon_{i+1}, g_{i+1}\right)\right. & =\delta\left(\mathcal{F} \epsilon_{i} \circ h ; h^{-1} \circ \mathcal{F} g_{i}\right) \\
& =\sup _{x \in N} d_{N}\left(x, h\left(\mathcal{F} \epsilon_{i}\left(\mathcal{F} g_{i}\left(h^{-1}(x)\right)\right)\right)\right) \\
& =\sup _{y \in \mathcal{F} N} d_{N}\left(h(y), h\left(\mathcal{F} \epsilon_{i}\left(\mathcal{F} g_{i}(y)\right)\right)\right) \\
& =\sup _{y \in \mathcal{F} N} d\left(y, \mathcal{F} \epsilon_{i} \mathcal{F} g_{i}(y)\right) \\
& =\delta\left(\mathcal{F} \epsilon_{i}, \mathcal{F} g_{i}\right) \\
& \leq \epsilon \cdot \delta\left(\epsilon_{i}, g_{i}\right) .
\end{aligned}
$$

Hence $\delta\left(\left(\epsilon_{i}, g_{i}\right)\right) \leq \epsilon^{i-1}$ with $\epsilon<1$ and by Remark $3 \tilde{d}_{N, \epsilon_{i}}\left(\mathcal{F}^{i} \emptyset, N\right)$ converges to zero. Hence $N$ is the limes of the $\mathcal{F}^{i} \emptyset$ in $\wp_{c}(N), i \geq 1$. As $N$ was chosen arbitrarily, as the embedding sequence $\lambda_{i}: \mathcal{F}^{i} \emptyset \rightarrow \mathcal{F}^{i+1} \emptyset$ was constructed independently of 
$N$, and as the embeddings $\epsilon_{i}: \mathcal{F}^{i} \emptyset \rightarrow N$ are compatible with the $\lambda_{i}$, we conclude that all fixed points coincide up to isometry.

\section{$\underline{\text { Remark } 4}$}

Let $\mathcal{F}_{1}: \mathrm{C} \rightarrow \mathrm{C}$ be a contracting functor, let $\mathcal{F}_{2}: \mathrm{C} \rightarrow \mathrm{C}$ be a functor such that

$$
\delta\left(\mathcal{F}_{2} \iota\right) \leq \delta(\iota)
$$

i.e. weakly contracting, then $\mathcal{F}_{1} \circ \mathcal{F}_{2}$ and $\mathcal{F}_{2} \circ \mathcal{F}_{1}$ are trivially contracting. Hence we may start with one contracting functor $\mathcal{F}_{1}: \mathrm{C} \rightarrow \mathrm{C}$, as e.g. the functor

$$
\mathcal{F}_{1}(X)=\left\{p_{0}\right\} \cup(A \times X) \quad A \text { a set }
$$

in [3], where the metric $\bar{d}$ on $A \times X$ is given by

$$
\bar{d}\left((a, x),\left(a^{\prime}, x^{\prime}\right)\right)= \begin{cases}1 & \text { if } a \neq a^{\prime} \\ \frac{1}{2} d\left(x, x^{\prime}\right) & \text { otherwise }\end{cases}
$$

and $d$ is the metric of $X$. Then we can construct a variety of functors with unique fixed points using various functors in the construction process that are just weakly contracting. 


\section{Conclusion}

We have shown that for a contracting functor $\mathcal{F}: \mathrm{C} \rightarrow \mathrm{C}$, there is a "minimal" fixed point. This result fits in between the existence proof of [1] and their uniqueness result. The uniqueness result of [1] is based on a change of category (instead of $\mathrm{C}$ the category of complete metric spaces with a base point is considered) together with the property of hom-contractivity, see [1], expressing a condition of $\mathcal{F}$ with respect to morphisms. In contrast to this our approach to guarantee uniqueness is by satisfying that the functor $\mathcal{F}$ applied to the empty set yields a one-element space. When we look at the original paper of [3] and review the construction of spaces, i.e.

$$
\begin{aligned}
P_{0} & =\left\{p_{0}\right\} \\
P_{i+1} & =\left\{p_{0}\right\} \cup A \rightarrow \wp_{c}\left(B \times P_{i}\right) \\
& =\mathcal{F}\left(P_{i}\right),
\end{aligned}
$$

we see that we can always interprete $P_{0}$ as $\mathcal{F} \emptyset$, i.e. the condition for uniqueness is satisfied. In other words, we may interprete the 'nil' process as the result of applying $\mathcal{F}$ to the empty space. The relation between hom-contractivity and the condition $|\mathcal{F} \emptyset|=1$ will be dealt with separately in a forthcoming paper. 


\section{References}

1. America, P., Rutten, J. J. M. M.: Solving reflexive domain equations in a category of complete metric spaces. Report CS-R8709. Centre for Mathematics and Computer Science Amsterdam (1987). Also in: 3rd Workshop on Mathematical Foundations of Programming Languages. Springer Lecture Notes in Computer Science (1988)

2. Arbib, M., Manes, E.: Structures and Functors. Academic Press. New York (1975)

3. de Bakker, J.W., Zucker, J.: Processes and the denotational semantics of concurrency. Information and Control 54, 70-120 (1982)

4. Engelking, R.: General Topology. Polish Scientific Publishers, Warsaw (1977)

5. Francez, N., Hoare, C. A. R., Lehman, D., De Roever, W. P.: Semantics of nondeterminism, concurrency and communication. J. Comp. Sys. Sci. 19, 290-308 (1979)

6. Golson, W. G., Rounds, W. C.: Connections between two theories of concurrency. Information and Control 57, 102-124 (1983)

7. Hahn, H.: Reelle Funktionen. Chelsea, New York (1948)

8. Hoare, C. A. R.: Communicating sequential processes. Comm. ACM 21, 666-677 (1978)

9. Majster-Cederbaum, M. E., Zetzsche, F.: The relation between Hoare-like and de Bakker-style semantics for CSP. In preparation.

10. Majster-Cederbaum, M. E., Zetzsche, F.: Foundation for semantics in complete metric spaces. Technical report 1/1987 Reihe Informatik, Fakultät für Mathematik und Informatik der Universität Manmheim (1987). Submitted.

11. Nivat, M.: Infinite words, infinite trees, infinite computations. Foundations of Computer Science III.2 Mathematical Centre Trakt, No. 109 (1979)

12. Nivat, M.: Synchronisation of concurrent processes, in: "Formal language theory" (R. V. Book, Ed.) 429-454, Academic Press, New York (1980)

13. Scott, D. S.: Data types as lattices. SIAM J. of Computing, 5, 522-587 (1976)

14. Scott, D. S.: Continuous lattices. In: Toposes, algebraic geometry and logic. Proc. 1971 Dalhousie Conference. Lecture Notes in Math. 274, 97-136, Springer (1972)

15. Smyth, M. B.: Power domains. J. Comp. Sys. Sciences 16, 23-36 (1978)

16. Smyth, M. B., Plotkin, G. D.: The category-theoretic solution of recursive domain equations. SIAM J. of Computing 11, 761-781 (1982)

17. Zetzsche, F.: Untersuchung zweier denotationaler Semantikdefinitionen von CSP. $\mathrm{Ph}$. D. Thesis, Universität Mannheim (1987) 